Original

\title{
Evaluation of genial tubercle anatomy using cone beam computed tomography
}

\author{
Mehmet E. Kolsuz'1), Kaan Orhan ${ }^{1,2)}$, Burak Bilecenoglu'), \\ Bayram U. Sakul4), and Adnan Ozturk ${ }^{5}$
}

\begin{abstract}
1)Department of Dentomaxillofacial Radiology, Faculty of Dentistry, Ankara University, Ankara, Turkey
2)Department of Dentomaxillofacial Radiology, Faculty of Dentistry, Near East University, Mersin, Turkey

${ }^{3}$ Department of Anatomy, Faculty of Dentistry, Ankara University, Ankara, Turkey

4)Department of Anatomy, Faculty of Medicine, Medipol University, Istanbul, Turkey

5)Department of Oral and Maxillofacial Surgery, Faculty of Dentistry, Ankara University, Ankara, Turkey
\end{abstract}

(Received October 10, 2014; Accepted May 11, 2015)

\begin{abstract}
The purpose of this study was to characterize the anatomy of the genial tubercle using cone beam computed tomography (CBCT). The morphology and detailed anatomy of the genial tubercle were assessed retrospectively in 201 patients (101 females, 100 males) using CBCT images. The parameters examined were the height (GH) and width $(\mathrm{GW})$ of the genial tubercle, the distance from the lower incisors to the superior border of the tubercle (I-SGT), the distance from the inferior margin of the tubercle to the inferior margin of the mandible (IGM-IBM), and the anterior mandible thickness (AMT). Statistical analysis was performed to assess relationships among these parameters, gender, and orthodontic malocclusion $(P<\mathbf{0 . 0 5})$. The values obtained were GH 7.3-8.7 $\mathrm{mm}$, GW 7.9-9.2 mm, I-SGT 7.1-9.1 mm, IGM-IBM 8.3-10.1 mm, and AMT 14.0-16.2 mm. GH, GW, and I-SGT showed no significant differences between genders $(P>0.05)$. However, IGM-IBM was larger for class III than for class I and class II male patients, and larger than for class I female patients. AMT in class III patients was greater than in class I and II patients $(P<0.05)$. The use of CBCT, which employs less radiation, is important for dental professionals,
\end{abstract}

Correspondence to Dr. Mehmet Eray Kolsuz, Department of Dentomaxillofacial Radiology, Faculty of Dentistry, Ankara University, 06500 Besevler, Ankara, Turkey

Fax:+90-312-212-3954 E-mail: m_eraykolsuz@yahoo.com

doi.org/10.2334/josnusd.57.151

DN/JST.JSTAGE/josnusd/57.151 especially those performing surgery for obstructive sleep apnea (OSA), in order to avoid possible surgical complications. (J Oral Sci 57, 151-156, 2015)

Keywords: cone-beam CT; anatomy; genial tubercle; obstructive sleep apnea syndrome; surgery.

\section{Introduction}

Obstructive sleep apnea (OSA) can be defined as cessation of breathing during sleep because of mechanical obstruction such as retropositioning of the tongue in the airway, a large amount of tissue in the upper airway, or even a partially collapsed trachea. OSA is a common respiratory sleep disorder characterized by snoring and episodes of breathing cessation or absence of respiratory airflow during sleep, and despite respiratory effort $(1,2)$. Patients with OSA can be defined as having daytime sleepiness, which may be associated with hypertension, nocturnal hypoxemia, cerebrovascular pathology, and cardiac problems, especially arrhythmia (3). The pathophysiological factors associated with OSA are very complex, ranging from those pertaining to ventilatory control or sensory function to anatomical abnormalities that may promote upper airway collapse, including skeletal, soft tissue and pharyngeal muscle factors $(4,5)$. One specific anatomical location seems unlikely to be a major cause of upper airway collapse, and it is more probably a dynamic phenomenon involving several factors and locations $(5,6)$.

It has been suggested that the genioglossus muscle, 
which serves as dilator of the pharynx, may play a role in airway obstruction (7-10). The genioglossus muscle arises from the spine of the mentum and the genial tubercles, which are aligned posteriorly at the tongue base, and extends to the hyoid bone. The function of the genioglossus is to retract the tip as well as depress the dorsum of the tongue. The inferior fibers advance the hyoid anterosuperiorly. The attachments of the genial tubercle are intimately involved in the function and support of the tongue and its associated soft tissues $(7,11,12)$.

Several surgical approaches for advancing the mandible anteriorly in order to increase the hypopharyngeal airway space in patients with OSA have been reported $(10,12,13)$. This is done to position the tongue muscles anteriorly in order to decrease airway resistance. However, the anatomy and morphology of these muscles are not clearly defined in the literature (11-13).

Three-dimensional (3D) imaging has been used for diagnosis and treatment of various conditions in patients with maxillofacial deformities $(14,15)$. Such imaging has also been used for accurate identification of the genial tubercle in preparation for genioglossus advancement in the management of OSA (16). In this context, it is very important to define the anatomy and morphology of the genial tubercle in relation to the anterior teeth and margins of the mandible $(7,10,12,17,18)$.

To our knowledge, no previously reported study has examined the location and morphology of the genial tubercle using cone beam computed tomography (CBCT). Therefore, the purpose of the present study was to use CBCT to characterize the anatomy and morphology of the genial tubercle in preparation for OSA surgery in a Turkish population with various types of malocclusion.

\section{Materials and Methods}

Our study group comprised 201 patients (101 [50.2\%] females, 109 [49.8\%] males) who had undergone CBCT imaging at the faculty of dentistry between January 2008 and January 2013. The patients ranged in age from 20-76 $\mathrm{yr}$ with a mean age of $40.3 \mathrm{yr}$. The sample was constituted as Law of Large Numbers and all appropriate CBCT images from the archive were included in the study. The study protocol conformed to the Declaration of Helsinki, including all amendments and revisions. The consent forms were reviewed and approved by the faculty board (\#HAH-F10/2008), and the forms were signed by the patients or their legal representatives. The patients also gave consent for publication of their photos/radiographs. The CBCT images had been taken for implant evaluation, surgery for impacted teeth, OSA, and pre-orthodontic or pre-prosthetic evaluation. Any patients with bone disease (especially osteoporosis), drug-related bone changes, skeletal asymmetries, congenital disorders or syndromes, or who had already undergone surgery were excluded. Low-quality images that did not allow sufficient visualization of bone borders, or those with artefacts or insufficient magnification were also omitted.

A Newtom 3G (Quantitative Radiology s.r.l., Verona, Italy; $120 \mathrm{kVP}, 3-5 \mathrm{~mA}$, slice thickness $0.3 \mathrm{~mm}$ ) was used for CBCT imaging. The machine can switch automatically in terms of $\mathrm{kV}$ and $\mathrm{mA}$, allowing a decrease of exposure of up to $40 \%$, and yields 12-bit gray scale images with a voxel size of $0.4 \mathrm{~mm}^{3}$. In order to standardize the scans, patients were positioned at the same axial view level based on their bilateral zygomatic sutures. The evaluations were performed using a LCD medical display with a $1,980 \times 1,600$ resolution dot pitch operated at 12-bit resolution (NEC MultiSync MD215MG, Munich, Germany).

Two dentomaxillofacial radiologists with 16 years and 7 years of experiences (K.O., M.E.K) reviewed the $\mathrm{CBCT}$ images. Each image was reconstructed using multiple projection (MPR). The infraorbital foramina on both sites were confirmed to correspond in coronal view. Then sagittal and axial MPR images clearly depicting the genial tubercles were chosen for measurement using the software provided with the CBCT device (NNT 4.6, QR Verona, Italy). The parameters measured were the height $(\mathrm{GH})$ and width $(\mathrm{GW})$ of the genial tubercle, the distance from the inferior margin of the genial tubercle to the inferior margin of the mandible (IGM-IBM), and the anterior mandible thickness (AMT) (Fig. 1a). Moreover, the apices of the lower incisors were clearly determined after evaluation of consecutive cross-sections, and then the distance from the lower incisors to the superior border of the tubercle (I-SGT) was calculated (Fig. 1b).

Genial tubercle width $(\mathrm{GW})$ was also measured from axial images obtained by CBCT. In order to correctly define the genial tubercle from axial images, consecutive images in which the genial tubercle was clearly identified were examined. Then, measurement of GW was performed from the two consecutive axial slices, and the mean value was noted as the final width of the genial tubercle (Fig. 2).

Malocclusions in patients were classed into three groups according to the ANB angle and sagittal interjawbase relationship. CBCT-generated cephalograms were used for malocclusion analysis. The three groups were: Class I: Overjet 2-4 mm; ANB angle $0^{\circ}-4^{\circ} ; \mathrm{SNA}=82 \pm$ 2 ; $\mathrm{SNB}=80 \pm 2$

Class II: Overjet $\geq 5 \mathrm{~mm}$; ANB angle $>4^{\circ}$; $\mathrm{SNA}>82$; $\mathrm{SNB}$ $<78$ 


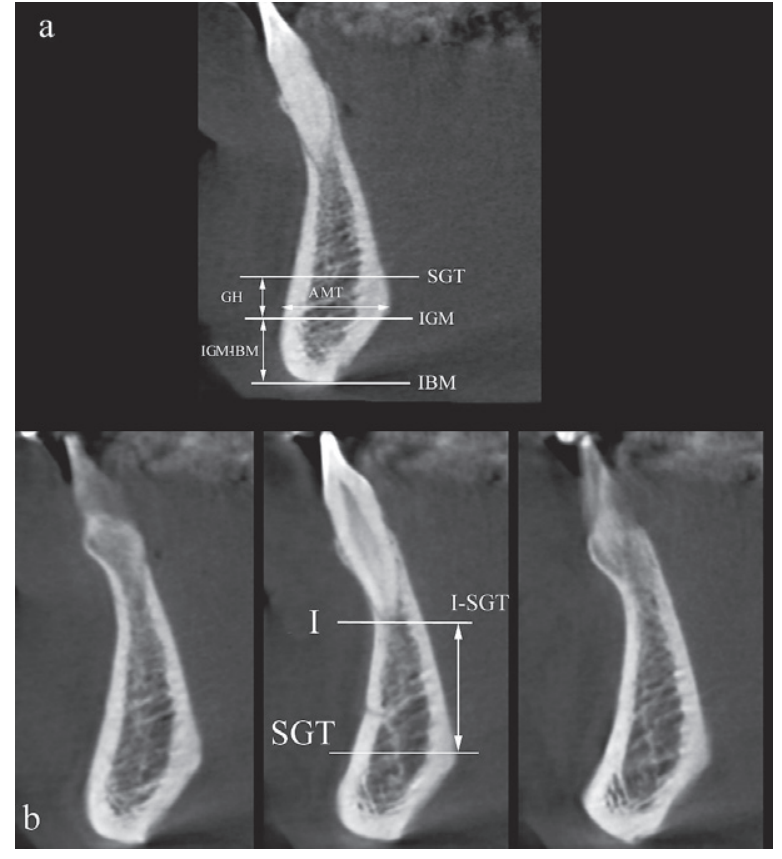

Fig. 1 (a) CBCT scan showing the parameters evaluated: thickness of the anterior mandible (AMT), genial tubercle height (GH; distance from the superior border [SGT] to the inferior border of the genial tubercle [IGM]), and distance from the IGM to the inferior border of the mandible (IBM) (IGM-IBM). (b) Consecutive cross-sectional images help to determine the apices of the incisors, which allow measurement of the I-SGT (distance from the apices of the lower incisor [LI] to the superior border of the genial tubercle [SGT]).

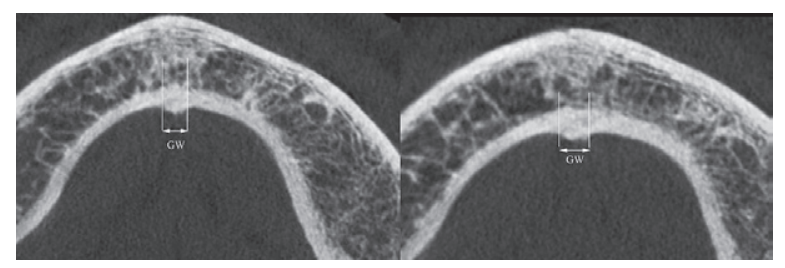

Fig. 2 Consecutive axial images for identification of the genial tubercle and measurement of the GW were obtained using two consecutive axial slices, and the mean of this measurement was taken as the final width of the genial tubercle.

Class III: Negative overjet; ANB angle $<0^{\circ}$; $\mathrm{SNA}<80$; $\mathrm{SNB}>84$.

Prior to the study, a calibration session was conducted in order to reach a consensus about the anatomic region of the genial tubercles and to recognize the surrounding structures.

For this purpose, $50 \mathrm{CBCT}$ images that did not form part of the study group were used that allowed the observers to understand the definitions of the anatomical landmarks and to become familiar with the measurements.

The measurements were performed by two observers three times, and the mean values of these measurements
Table 1 Inter-observer consistency of measurements

\begin{tabular}{lcc}
\hline \multirow{2}{*}{ Measurements } & \multicolumn{2}{c}{ Observer $1-2$} \\
\cline { 2 - 3 } & ICC & CV $(\%)$ \\
\hline GH & 0.815 & 2.16 \\
GW & 0.850 & 2.08 \\
I-SGT & 0.900 & 1.76 \\
Vertical outer diameter & 0.910 & 1.80 \\
IGM-IBM & 0.924 & 1.88 \\
AMT & 0.911 & 1.86 \\
\hline
\end{tabular}

$\mathrm{CV}$, coefficient of variation; ICC, intraclass correlation coefficient.

were calculated as the final data. The observers also performed two measurement sessions 2 weeks apart in order to detect intra-observer variability.

Intra- and inter-examiner reliability was confirmed. Wilcoxon matched-pairs signed rank test was used to assess intra-observer reliability, whereas intraclass correlation coefficient (ICC) and the coefficient of variation $(\mathrm{CV})$ were used to assess inter-observer reliability. Values for the ICC ranged from 0 to 1 . ICC values higher than 0.75 indicate good reliability, whereas a low CV demonstrates the precision error as an indicator of reproducibility (19). Pearson chi-squared test and ANOVA were performed (SPSS 17, Chicago, IL, USA) to assess the significance of inter-group differences in age, gender, and orthodontic malocclusion $(P<0.05)$.

\section{Results}

CBCT evaluation and measurements revealed no intraobserver variance for either of the observers $(P>0.05)$. Overall measurement consistency for observer 1 was rated at $86.7 \%$ and that for observer 2 was $89.2 \%$. All measurements were highly correlated for both observers, and no significant difference was evident for repeated measurements by the observers $(P>0.05)$.

ICCs between the two observers ranged from 0.815 to 0.924 (Table 1). There was high inter-observer agreement, while a high ICC and low CV demonstrated that the procedure was standardized between the evaluations and measurements performed by the observers. No significant differences were seen in any of the variables $(P>0.05)$.

The mean values of the measured parameters were 7.3-8.7 $\mathrm{mm}$ for $\mathrm{GH}, 7.9-9.2 \mathrm{~mm}$ for $\mathrm{GW}, 7.1-9.1 \mathrm{~mm}$ for I-SGT, 8.3-10.1 mm for IGM-IBM, and 14.0-16.2 mm for AMT. In all groups, GH was close to GW. GH, GW, and I-SGT showed no significant differences among the groups $(P>0.05)$. IGM-IBM was higher for class III than for class I and class II male patients, and higher than the value for class I female patients $(P<0.05)$. AMT in class III patients was denser than in class I and II patients $(P<0.05)$ (Table 2). 
Table 2 The mean values of various parameters according to gender and type of malocclusion

\begin{tabular}{|c|c|c|c|c|c|c|c|}
\hline \multirow{2}{*}{ Measurements } & \multicolumn{2}{|c|}{ Class I } & \multicolumn{2}{|c|}{ Class II } & \multicolumn{2}{|c|}{ Class III } & \multirow{2}{*}{$P$ value } \\
\hline & Male & Female & Male & Female & Male & Female & \\
\hline GH & $7.7 \pm 1.8$ & $7.3 \pm 1.6$ & $7.9 \pm 1.9$ & $7.6 \pm 1.8$ & $8.7 \pm 2.1$ & $8.2 \pm 1.8$ & $>0.05$ \\
\hline GW & $8.1 \pm 1.2$ & $7.9 \pm 1.1$ & $8.3 \pm 1.2$ & $8.2 \pm 1.1$ & $9.2 \pm 1.2$ & $8.8 \pm 1.0$ & $>0.05$ \\
\hline I-SGT & $8.1 \pm 1.7$ & $7.7 \pm 1.8$ & $7.5 \pm 1.8$ & $7.1 \pm 1.5$ & $9.1 \pm 2.2$ & $8.7 \pm 1.9$ & $>0.05$ \\
\hline IGM-IBM & $8.3 \pm 2.3^{\mathrm{a}, \mathrm{c}}$ & $8.4 \pm 2.3^{\mathrm{b}}$ & $9.2 \pm 2.1^{\mathrm{a}}$ & $9.0 \pm 2.0$ & $10.1 \pm 2.2^{\mathrm{a}}$ & $9.4 \pm 2.2^{\mathrm{b}, \mathrm{c}}$ & $<0.05$ \\
\hline AMT & $14.8 \pm 2.9^{\mathrm{a}, \mathrm{c}}$ & $14.0 \pm 2.4^{\mathrm{b}, \mathrm{d}}$ & $15.4 \pm 2.7^{\mathrm{a}, \mathrm{b}}$ & $15.0 \pm 2.4^{b}$ & $16.2 \pm 2.5^{\mathrm{a}, \mathrm{b}, \mathrm{c}, \mathrm{d}}$ & $16.0 \pm 2.5^{\mathrm{b}}$ & $<0.05$ \\
\hline
\end{tabular}

* same letters indicate statistical significance.

\section{Discussion}

Surgical intervention is indicated for patients with OSA when conservative therapies are not effective or cannot be tolerated. The correct surgical approach is established for the obstructed site in the upper airway in each individual patient. Soft tissue surgery, such as uvulopalatopharyngoplasty, is used for patients with solely velopharyngeal obstruction $(9,18)$.

Mandibular advancement surgery has been advocated for surgical management of OSA, especially in patients with airway resistance and obstruction in the hypopharynx and base of the tongue. The aim of this surgery is to advance the genial tubercle to achieve posterior relocation of the tongue during sleep $(1,8,9)$.

Genioglossus advancement surgery has four aims: 1) to avoid damage to the roots of anterior teeth, 2) to integrate the fibers of the muscle, 3) to prevent mandibular fracture, and 4) to maximize the effectiveness of genioglossus advancement (10). In the first step of the operation, the horizontal osteotomy should be placed at least $5 \mathrm{~mm}$ away from the anterior teeth $(7,12)$. Determination of the I-SGT distance is crucial in the first step, as this value varies among populations. A study of 10 European cadavers recorded a distance of $11.8 \mathrm{~mm}$ (7), while other studies of Chinese cadavers recorded a distance of $15.6 \mathrm{~mm}$ in males and $9.4 \mathrm{~mm}$ in females $(9,20)$. In our present study, the I-SGT distance ranged from 7.1 to $9.1 \mathrm{~mm}$, which was in line with the results of Mintz et al. (21), who found a mean I-SGT of $6.5 \mathrm{~mm}$ in 41 human skulls, with a range of 1-14 $\mathrm{mm}$. In their study, $35 \%$ of the genial tubercles were $5 \mathrm{~mm}$ from the lower incisors. Our results also indicated that $18 \%(36 / 201)$ of our patients had an I-SGT distance of less than $5 \mathrm{~mm}$.

The present retrospective study showed that the genial tubercle varied in its position, morphology and dimensions in the study group. The anterior region of the mandible also varied in its morphology according to gender and type of malocclusion. Previous studies $(7,8,11)$ have investigated only class I or class II skeletal types, which are typical of patients with OSA $(9,22)$.
Wang et al. (9) divided their study patients into Class I and Class II groups, and obtained results similar to ours. However, we also included Class III patients in whom the values for various parameters were higher than in the other groups. We also found that the anterior mandible thickness gradually increased from Class I to Class III, being significantly greater in the latter $(P<0.05)$.

Eighteen of our study patients in whom the I-SGT distance was below $5 \mathrm{~mm}$ were in the Class II group $(18 / 201)$. Very few studies have compared Angle Class correlations with the detailed morphology of the genial tubercle, and even fewer have determined airway dimensions. Kirjavainen and Kirjavainen (23) reported that subjects with Class II malocclusion showed a narrower oro- and hypopharyn-geal space than subjects with Class I and normal occlusion. They also emphasized vari-ability in vertical dimensions and its impact. In our opinion, Class II subjects scheduled for airway or OSA surgery should be examined carefully before any interventions. Overall, the results of the present study confirm previous data indicating that a horizontal cut of $5 \mathrm{~mm}$ may also be safe in this particular population.

Our present study showed that the mean AMT was 14.2 to $16.2 \mathrm{~mm}$, which was similar but slightly higher than values obtained in previous studies $(8,11,18,21)$. Our results indicated that Class III patients have a thicker AMT than Class I and Class II patients. Wang et al. (9) reported that Class I male patients had denser mandibles than Class II female patients, thus being more amenable to surgery. Based on the results for our population, it can be easily suggested that Class III male and female patients are better suited to genioglossus advancement surgery.

The second step of OSA surgery is a vertical cut indicated by GW. Our present GW values were between 7.9 and $9.2 \mathrm{~mm}$, being similar to those obtained by Wang et al. (9) and coinciding with those $(6.0 \mathrm{~mm})$ reported by Mintz et al. (21). This may have been due to the particular populations studied. The horizontal cut in a box osteomy should be more than $10 \mathrm{~mm}$ above the inferior border 
of the mandible to prevent mandibular fracture (12). A mean IGM-IBM of 8.3-10.1 $\mathrm{mm}$ can be interpreted as a lack of sufficient bone for OSA surgery. The values found in this study were in line with those of Wang et al. study (9) but less than those in another study (20). Class I subjects in our study had the lowest IGM-IBM value, and thus care would have been needed before surgery in this particular population.

In this study, we used CBCT for evaluation of anterior mandible morphology and the genial tubercles. Compared with standard medical CT scans, dental CBCT has been recommended as a dose-sparing technique for imaging of anatomical landmarks before surgical procedures. The effective dose (ICRP 2007) from a standard dental protocol scan using traditional CT is 1.5 to 12.3 times greater than that for comparable medium-FOV dental CBCT scans. CBCT image quality has also been found to be equivalent to that of traditional $\mathrm{CT}$ for visualization of maxillofacial structures (24-26).

Special attention should be paid to the anterior mandible in patients scheduled for OSA surgery. CBCT has distinct advantages over MDCT in terms of a lower radiation dose and superior resolution, as well as its noninvasiveness and allowing import and export of individualized, overlap-free reconstructions (27).

Although CT is advantageous in facilitating a soft tissue window (muscles etc.), it is still difficult to analyze complex anatomy on the basis of CT imaging alone $(28,29)$. CT has been of great value for evaluation of anatomical structures, but despite the fact it can yield very thin adjacent slices, it is not yet a perfect technique. As our present study has shown, CBCT has a smaller voxel size and high spatial resolution for detecting small anatomical structures, with less exposure to ionizing radiation.

One limitation of this particular study was that it was a population-based study involving only Turkish patients. The incidence of malocclusion types may vary in different populations, and several studies (30-33) have shown that the distribution of malocclusion is affected by the ethnic and cultural characteristics of the study sample (30). However, Class III malocclusion is usually the type that is encountered least frequently in sagittal projections $(32,33)$. Epidemiological studies have found that patients with Class III malocclusion accounted for $10-12 \%$ in Turkish orthodontic patients (30), compared with $4.5 \%$ of 532 school children in India aged 7-13 yr (33). Therefore, further comparative studies of different populations seem warranted. Moreover, before OSA surgery, there is a need to carefully evaluate the type of malocclusion using both clinical and radiological techniques.
Another limitation of this study is that it was based on radiological data. Among previous anatomical cadaver studies $(8,32)$, Silverstein (8) concluded that the genioglossus muscle is broader than stated in classical anatomical texts. Therefore, comparison of actual anatomy with radiological data would be valuable, and further such compative studies would be warranted.

In summary, precise characterization of genial tubercle anatomy is crucial before OSA surgery. Clinicians need to exercise care during OSA surgery to avoid possible complications, paying special attention to patient gender and malocclusion, using CBCT to minimize the degree of radiation exposure.

\section{Conflict of interest}

The authors declare no conflict of interest.

\section{References}

1. Prinsell JR (1999) Maxillomandibular advancement surgery in a site-specific treatment approach for obstructive sleep apnea in 50 consecutive patients. Chest 116, 1519-1529.

2. Madani M, Madani F (2007) Definitions, abbreviations, and acronyms of sleep apnea. Atlas Oral Maxillofac Surg Clin North Am 15, 69-80.

3. Hendler BH, Costello BJ, Silverstein K, Yen D, Goldberg A (2001) A protocol for uvulopalatopharyngoplasty, mortised genioplasty, and maxillomandibular advancement in patients with obstructive sleep apnea: an analysis of 40 cases. J Oral Maxillofac Surg 59, 892-897.

4. Badr MS (1998) Pathophysiology of upper airway obstruction during sleep. Clin Chest Med 19, 21-32.

5. Azagra-Calero E, Espinar-Escalona E, Barrera-Mora JM, Llamas-Carreras JM, Solano-Reina E (2012) Obstructive sleep apnea syndrome (OSAS). Review of the literature. Med Oral Patol Oral Cir Bucal 17, e925-929.

6. Braga A, Carboni LH, do Lago T, Küpper DS, Eckeli A, Valera FC (2013) Is uvulopalatopharyngoplasty still an option for the treatment of obstructive sleep apnea? Eur Arch Otorhinolaryngol 270, 549-554.

7. McAndrew BP, Strauss RA (2000) Delayed muscle detachment after genial tubercle advancement in a patient with obstructive sleep apnea. J Oral Maxillofac Surg 58, 10401043.

8. Silverstein K, Costello BJ, Giannakpoulos H, Hendler B (2000) Genioglossus muscle attachments: an anatomic analysis and the implications for genioglossus advancement. Oral Surg Oral Med Oral Pathol Oral Radiol Endod 90, 686-688.

9. Wang YC, Liao YF, Li HY, Chen YR (2012) Genial tubercle position and dimensions by cone-beam computerized tomography in a Taiwanese sample. Oral Surg Oral Med Oral Pathol Oral Radiol 113, e46-50.

10. Agarwal S, Gaurav I, Agarwal R, Ahluwalia KS (2013) Determination of genial tubercle position and dimensions using cone-beam computerised tomography. Indian J Med 
Specialities 4, 29-33.

11. Riley RW, Powell NB, Guilleminault C (1989) Inferior mandibular osteotomy and hyoid myotomy suspension for obstructive sleep apnea: a review of 55 patients. J Oral Maxillofac Surg 47, 159-164.

12. Li KK, Riley RW, Powell NB, Troell RJ (2001) Obstructive sleep apnea surgery: genioglossus advancement revisited. J Oral Maxillofac Surg 59, 1181-1184.

13. Troell RJ, Riley RW, Powell NB, Li K (1998) Surgical management of the hypopharyngeal airway in sleep disordered breathing. Otolaryngol Clin North Am 31, 979-1012.

14. Fatemitabar SA, Nikgoo A (2010) Multichannel computed tomography versus cone-beam computed tomography: linear accuracy of in vitro measurements of the maxilla for implant placement. Int J Oral Maxillofac Implants 25, 499-505.

15. Abramson Z, Susarla SM, Lawler M, Bouchard C, Troulis M, Kaban LB (2011) Three-dimensional computed tomographic airway analysis of patients with obstructive sleep apnea treated by maxillomandibular advancement. J Oral Maxillofac Surg 69, 677-686.

16. Hueman EM, Noujeim ME, Langlais RP, Prihoda TJ, Miller FR (2007) Accuracy of cone beam computed tomography in determining the location of the genial tubercle. Otolaryngol Head Neck Surg 137, 115-118.

17. Barbick MB, Dolwick MF (2009) Genial tubercle advancement for obstructive sleep apnea syndrome: a modification of design. J Oral Maxillofac Surg 67, 1767-1770.

18. Goodday R (2009) Diagnosis, treatment planning, and surgical correction of obstructive sleep apnea. J Oral Maxillofac Surg 67, 2183-2196.

19. Chang PC, Liang K, Lim JC, Chung MC, Chien LY (2013) A comparison of the thresholding strategies of micro-CT for periodontal bone loss: a pilot study. Dentomaxillofac Radiol 42, 66925194.

20. Yin SK, Yi HL, Lu WY, Guan J, Wu HM, Cao ZY et al. (2007) Anatomic and spiral computed tomographic study of the genial tubercles for genioglossus advancement. Otolaryngol Head Neck Surg 136, 632-637.

21. Mintz SM, Ettinger AC, Geist JR, Geist RY (1995) Anatomic relationship of the genial tubercles to the dentition as determined by cross-sectional tomography. J Oral Maxillofac Surg 53, 1324-1326.

22. Pirklbauer K, Russmueller G, Stiebellehner L, Nell C, Sinko
K, Millesi G et al. (2011) Maxillomandibular advancement for treatment of obstructive sleep apnea syndrome: a systematic review. J Oral Maxillofac Surg 69, e165-176.

23. Kirjavainen M, Kirjavainen T (2007) Upper airway dimensions in Class II malocclusion. Effects of headgear treatment. Angle Orthod 77, 1046-1053.

24. Liang X, Jacobs R, Lambrichts I, Vandewalle G (2007) Lingual foramina on the mandibular midline revisited: a macroanatomical study. Clin Anat 20, 246-251.

25. Periago DR, Scarfe WC, Moshiri M, Scheetz JP, Silveira AM, Farman AG (2008) Linear accuracy and reliability of cone beam CT derived 3-dimensional images constructed using an orthodontic volumetric rendering program. Angle Orthod 78, 387-395.

26. Ludlow JB, Gubler M, Cevidanes L, Mol A (2009) Precision of cephalometric landmark identification: cone-beam computed tomography vs conventional cephalometric views. Am J Orthod Dentofacial Orthop 136, 312.

27. Orhan K, Aksoy S, Bilecenoglu B, Sakul BU, Paksoy CS (2011) Evaluation of bifid mandibular canals with cone-beam computed tomography in a Turkish adult population: a retrospective study. Surg Radiol Anat 33, 501-507.

28. Himi T, Sakata M, Shintani T, Mitsuzawa H, Kamagata M, Satoh J et al. (2000) Middle ear imaging using virtual endoscopy and its application in patients with ossicular chain anomaly. ORL J Otorhinolaryngol Relat Spec 62, 316-320.

29. Dalchow CV, Knecht R, Grzyska U, Muenscher A (2013) Radiographic examination of patients with dehiscence of semicircular canals with digital volume tomography. Eur Arch Otorhinolaryngol 270, 511-519.

30. Sayin MO, Turkkahraman H (2004) Malocclusion and crowding in an orthodontically referred Turkish population. Angle Orthod 74, 635-639.

31. Gelgör IE, Karaman AI, Ercan E (2007) Prevalence of malocclusion among adolescents in central anatolia. Eur J Dent 1, 125-131.

32. Celikoglu M, Akpinar S, Yavuz I (2010) The pattern of malocclusion in a sample of orthodontic patients from Turkey. Med Oral Patol Oral Cir Bucal 15, e791-796.

33. Prabhakar RR, Saravanan R, Karthikeyan MK, Vishnuchandran C, Sudeepthi (2014) Prevalence of malocclusion and need for early orthodontic treatment in children. $\mathrm{J}$ Clin Diagn Res 8, ZC60-61. 